\title{
Constraints on the Unseen Galaxy Population from the Ly $\alpha$ Forest
}

\author{
Kenneth M. Lanzetta and Hsiao-Wen Chen \\ Department of Physics and Astronomy, State University of New York at \\ Stony Brook, Stony Brook, NY 11794-3800, U.S.A. \\ John K. Webb \\ School of Physics, University of New South Wales, Sydney 2052, NSW, \\ AUSTRALIA \\ Xavier Barcons \\ Instituto de Física de Cantabria (Consejo Superior de Investigaciones \\ Cientificas-Universidad de Cantabria), Facultad de Ciencias, 39005 \\ Santander, SPAIN
}

\begin{abstract}
Here we describe results of our attempt to determine what types of galaxies are responsible for the Ly $\alpha$ forest absorption systems, based on our ongoing imaging and spectroscopic survey of faint galaxies in fields of HST spectroscopic target QSOs. Our primary conclusions are that the bulk of the Ly $\alpha$ forest arises in more or less normal galaxies (that span the normal range of luminosity and morphology) and that any "unseen" low surface brightness galaxies are unlikely to contribute significantly to the luminosity density of the universe.
\end{abstract}

\section{Introduction}

Our analysis of the relationship between galaxies and Ly $\alpha$ absorption systems at redshifts $z<1$ has over the past few years led us to conclude that (1) most galaxies possess extended gaseous envelopes of $\approx 160 h^{-1} \mathrm{kpc}$ radius and (2) many or most Ly $\alpha$ absorption systems arise in extended gaseous envelopes of galaxies (e.g. Lanzetta et al. 1995; Barcons et al. 1995; Chen et al. 1998). These conclusions bear directly, of course, on questions concerning the nature and physical state of gaseous material at very large galactocentric distances. But what is of more immediate relevance to the topic of this meeting is that these conclusions also imply that the "forest" of Ly $\alpha$ absorption lines that are routinely observed in the spectra of background QSOs probe galaxies (rather than something else) to redshifts as large as $z \approx 5$.

The Ly $\alpha$ resonance transition is an extraordinarily sensitive tracer of very low column density material. Ordinary spectra of ordinary QSOs can easily detect neutral hydrogen column densities as low as $N \approx 5 \times 10^{13} \mathrm{~cm}^{-2}$, which is many orders of magnitude below the column densities probed by star light or $21 \mathrm{~cm}$ emission. In this sense, the Ly $\alpha$ forest represents a more or less complete 
inventory of the baryonic constituents of the universe. Galaxies that are difficult to detect by "ordinary" means (i.e. by means of star light or $21 \mathrm{~cm}$ emission) are easy to detect by means of Ly $\alpha$ absorption lines, so it remains to establish what portion of the Ly $\alpha$ forest can be attributed to "normal" galaxies that are represented by normal galaxy luminosity functions in order to determine what portion of the Ly $\alpha$ forest is left over for "unseen" galaxies that are not represented by normal galaxy luminosity functions.

Here we describe the results of our attempt to determine what types of galaxies are responsible for the Ly $\alpha$ forest absorption systems, based on our ongoing imaging and spectroscopic survey of faint galaxies in fields of Hubble Space Telescope (HST) spectroscopic target QSOs. Using new observations of the galaxies of the survey, we have sought to establish just what factors play a role in determining the gaseous extent of galaxies. Our primary conclusions are that the bulk of the $\operatorname{Ly} \alpha$ forest arises in more or less normal galaxies (that span the normal range of luminosity and morphology) and that any "unseen" low surface brightness galaxies are unlikely to contribute significantly to the luminosity density of the universe. More stringent conclusions along these lines are within reach, requiring only further observations and analysis. Throughout we adopt a standard Friedmann cosmological model of deceleration parameter $q_{0}=0.5$ and Hubble constant $H_{0}=100 \mathrm{~h} \mathrm{~km} \mathrm{~s}^{-1} \mathrm{Mpc}^{-1}$.

\section{Imaging and Spectroscopic Survey}

Over the past several years, we have been conducting an ongoing imaging and spectroscopic survey of faint galaxies in fields of HST spectroscopic target QSOs. The goal of the survey is to determine the gaseous extent of galaxies and the origin of $L y \alpha$ absorption systems by directly comparing the redshifts of galaxies and absorbers identified along common lines of sight. The observations have been and will be described elsewhere (e.g. Lanzetta et al. 1995; Chen et al. 1998), but in summary the observations consist of (1) optical images and spectroscopy of objects in the fields of the QSOs, obtained with various telescopes and from the literature, and (2) ultraviolet spectroscopy of the QSOs, obtained with the HST using the Faint Object Spectrograph (FOS) and accessed through the HST archive. The optical images and spectroscopy are used to identify and measure galaxy redshifts and impact parameters, and the ultraviolet spectroscopy is used to identify and measure absorber redshifts and equivalent widths.

A total of 352 galaxies and 230 absorbers toward 24 fields are included into the current analysis. The galaxies and absorbers are "matched" or "associated" using quantitative criteria that are set by the galaxy-absorber cross-correlation function $\xi_{\mathrm{ga}}(v, \rho)$, as it depends on the line-of-sight velocity separation $v$ and the transverse impact parameter separation $\rho$. (Here we adopt the galaxy-absorber cross-correlation function measured previously by Lanzetta et al. 1997 on the basis of 3126 galaxy and absorber pairs.) In this way, "physical" galaxy and absorber pairs are quantitatively distinguished from "correlated" and "random" galaxy and absorber pairs. Galaxies and absorbers within $3000 \mathrm{~km} \mathrm{~s}^{-1}$ of the QSOs are excluded in order to focus the analysis on the "intervening" population.

Results of the survey are illustrated schematically in Figure 1. Figure shows in the top panel an image of the field surrounding 0454-2203 and in the bottom 


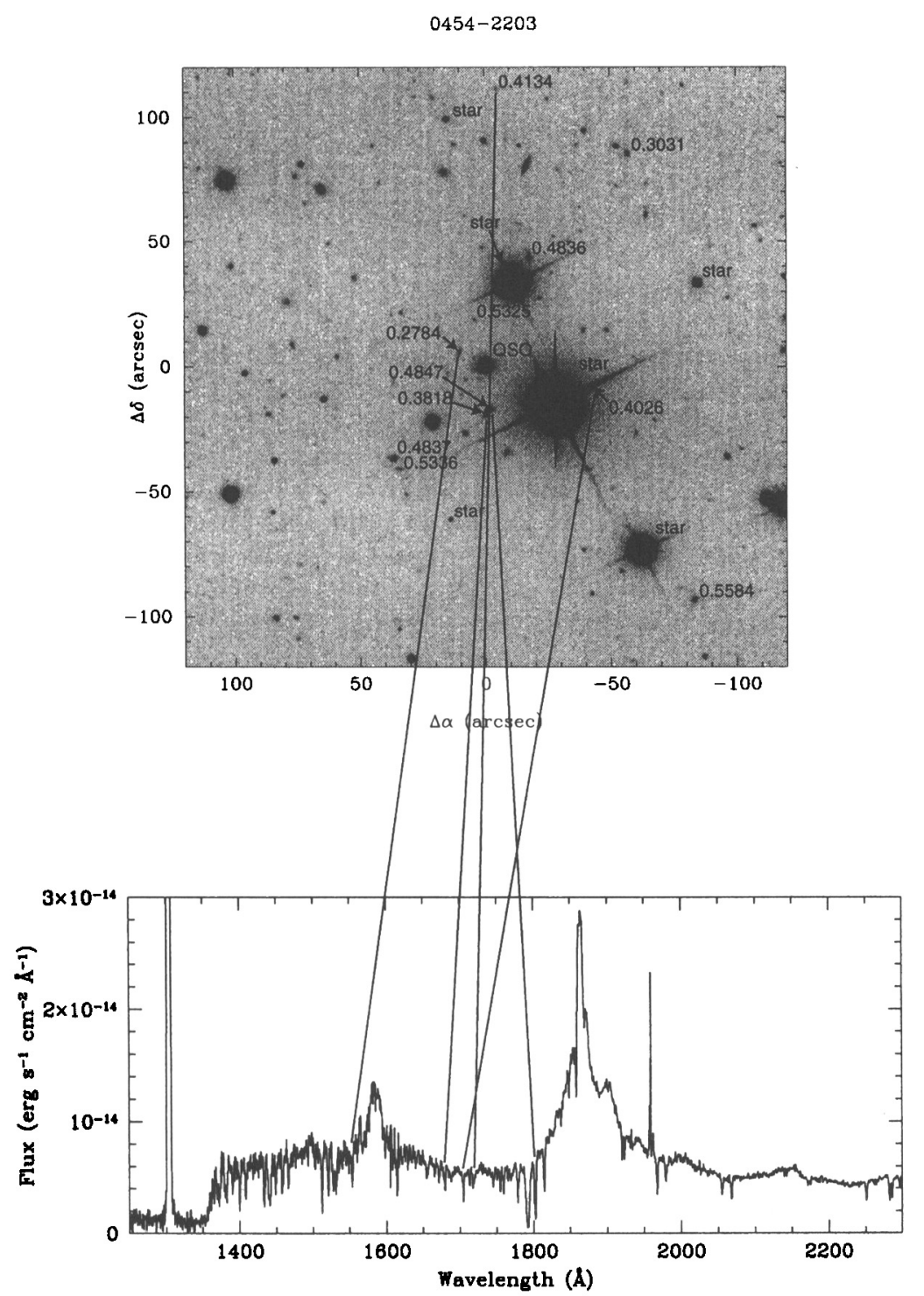

Figure 1. Schematic illustration of results of the survey. Top panel shows an image of the field surrounding 0454-2203. Bottom panel shows a spectrum of 0454-2203. Redshifts of the various faint galaxies so far identified by the survey are indicated in the top panel, and Ly $\alpha$ absorption lines matched with galaxies so far identified by the survey are indicated in the bottom panel. Redshift of $0454-2203$ is $z=0.54$, and the most prominent emission line in the spectrum is Ly $\alpha$. 
panel a spectrum of 0454-2203. Redshifts of the various faint galaxies so far identified by the survey are indicated in the top panel, and Ly $\alpha$ absorption lines matched with galaxies so far identified by the survey are indicated in the bottom panel. All matched galaxy and absorber pairs indicated in Figure 1 have cross-correlation amplitudes satisfying $\xi_{\mathrm{ga}}(v, \rho)>1$.

\section{Gaseous Extent of Galaxies}

One of the most striking results of the survey is that there exists a distinctive anti-correlation between Ly $\alpha$ absorption equivalent width $W$ and galaxy impact parameter $\rho$. In particular, galaxies at impact parameters less than $\approx 160 \mathrm{~h}^{-1}$ $\mathrm{kpc}$ are almost always associated with corresponding Ly $\alpha$ absorption systems while galaxies at impact parameters greater than $\approx 160 \mathrm{~h}^{-1} \mathrm{kpc}$ are almost never associated with corresponding Ly $\alpha$ absorption systems. The anti-correlation is statistically highly significant and persists even when various subsamples (e.g. absorption systems that exhibit metal absorption lines, or absorption systems that exhibit very strong Ly $\alpha$ absorption lines) are arbitrarily removed from the analysis. On the basis of this result, we conclude that galaxies are surrounded by extended gaseous envelopes of $\approx 160 h^{-1} \mathrm{kpc}$ radius. The anti-correlation between Ly $\alpha$ absorption equivalent width and galaxy impact parameter is shown in the top panel of Figure 2.

Yet it is clear from Figure 2 that the scatter about the mean relationship between $\operatorname{Ly} \alpha$ absorption equivalent width and galaxy impact parameter is quite substantial. Evidently the amount of gas encountered along the line of sight depends on other factors besides galaxy impact parameter, including perhaps galaxy luminosity, size, or morphological type; the geometry of the impact (e.g. if tenuous gas is distributed around galaxies in flattened disks rather than in spherical halos); or disturbed morphologies or the presence of close companions (e.g. if tenuous gas is distributed around galaxies as a result of interactions). To determine these other factors, we initiated a program to obtain and analyze HST Wide Field Planetary Camera 2 (WFPC) images of galaxies identified in the survey. These observations were obtained (and are being obtained) in HST Cycles 5 and 6 .

Using the WFPC2 images together with existing spectroscopic observations, we measured properties of galaxies identified in the survey, including rest-frame $B$-band luminosity $L_{B}$, effective radius $r_{e}$, average surface brightness $\langle\mu\rangle$, diskto-bulge ratio $D / B$, redshift $z$, and inclination and orientation angles. We then applied multivariate analysis techniques to search for a "fundamental surface" in the multidimensional space that is spanned by various combinations of the measurements. Initial results of the analysis are described by Chen et al. (1998).

The primary result of the analysis is that the amount of gas encountered along the line of sight depends on the galaxy impact parameter and $B$-band luminosity but does not depend strongly on the galaxy average surface brightness, disk-to-bulge ratio, or redshift. Spherical halos cannot be distinguished from flattened disks on the basis of the current observations, and there is no evidence that galaxy interactions play an important role in distributing tenuous gas around galaxies in most cases. These results are presented in the bottom panel of Figure 2 and in Figure 3. The bottom panel of Figure 2 shows the 

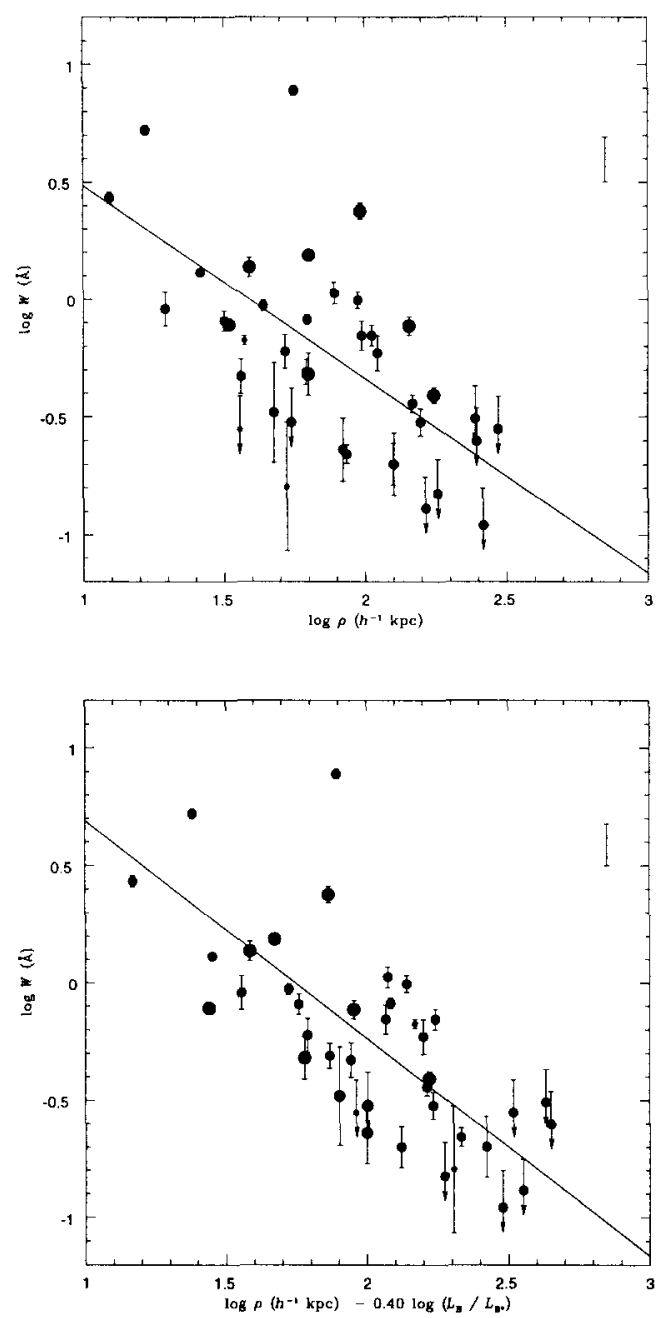

Figure 2. Top panel: Anti-correlation between Ly $\alpha$ absorption equivalent width $W$ and galaxy impact parameter $\rho$. Bottom panel: Anticorrelation between Ly $\alpha$ absorption equivalent width $W$ and galaxy impact parameter $\rho$ as "corrected" by the best-fit scaling with galaxy $B$-band luminosity $L_{B}$. Symbol size indicates galaxy luminosity (larger symbols indicate larger luminosities) and symbol type indicates galaxy morphological type (circles for elliptical and S0 galaxies, triangles for early-type spiral galaxies, and squares for late-type spiral galaxies). The error bars in the upper right corners indicate "cosmic" scatter. Both panels are based on galaxies for which HST WFPC2 images have already been obtained. 

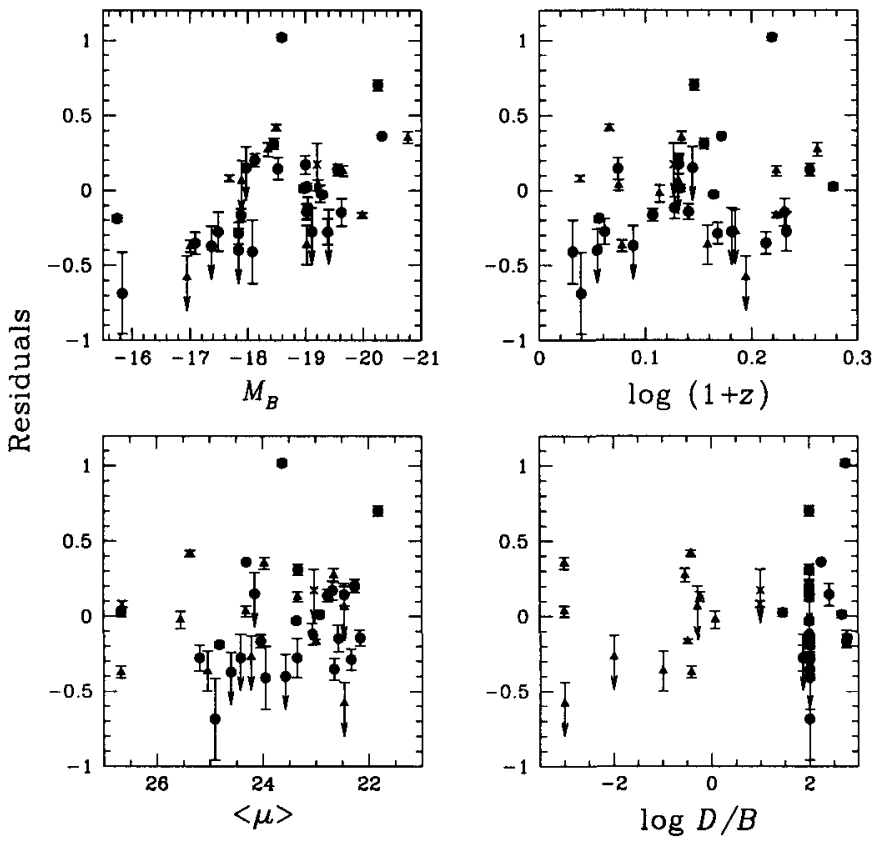

Figure 3. Residuals with respect to the best-fit Ly $\alpha$ absorption equivalent width versus galaxy impact parameter relation as functions of galaxy $B$-band absolute magnitude $M_{B}$, redshift $\log (1+z)$, average surface brightness $\langle\mu\rangle$, and disk-to-bulge ratio $\log D / B$. Symbols are as for Figure 2.

anti-correlation between $\mathrm{Ly} \alpha$ absorption equivalent width and galaxy impact as "corrected" by the best-fit scaling with galaxy $B$-band luminosity. Figure 3 shows the residuals with respect to the best-fit Ly $\alpha$ absorption equivalent width versus galaxy impact parameter relation as functions of galaxy $B$-band absolute magnitude $M_{B}$, redshift $\log (1+z)$, average surface brightness $\langle\mu\rangle$, and disk-to-bulge ratio $\log D / B$.

We ascribe two especially important implications to the remarkably tight anti-correlation between Ly $\alpha$ absorption equivalent width and galaxy impact as corrected by the best-fit scaling with galaxy $B$-band luminosity: First, it indicates that we have generally matched the appropriate galaxies with the appropriate absorption systems. Second, it indicates that galaxies are surrounded by distinct gaseous "envelopes" and that these envelopes are tightly associated with the individual galaxies, rather than loosely associated with the "large-scale environments" of the individual galaxies, e.g. as inter-group or inter-cluster gas. The result that the amount of gas encountered along the line of sight does not depend strongly on the galaxy average surface brightness, disk-to-bulge ratio, or redshift apparently indicates that extended gaseous envelopes are a common 
and generic feature of galaxies spanning a wide range of luminosity and morphological type and therefore that the Ly $\alpha$ forest traces a representative portion of the galaxy population.

The scaling relationship between galaxy gaseous radius $R$ and galaxy $B$ band luminosity $L_{B}$ is well described by

$$
\frac{R}{R_{*}}=\left(\frac{L_{B}}{L_{B_{*}}}\right)
$$

This relationship is analogous to the Holmberg relationship between galaxy stellar radius and galaxy $B$-band luminosity. Based on a sample that is slightly larger than the sample analyzed by Chen et al. (1998), the best-fit parameter estimates are (Chen et al. 1999, in preparation)

$$
t=0.40 \pm 0.09
$$

and

$$
R_{*}=190 \pm 34 h^{-1} \mathrm{kpc},
$$

which applies for Ly $\alpha$ absorption equivalent widths satisfying $W>0.32 \AA$. The most important implication of the scaling relationship of equation (1) is that it provides, for the first time, a means of quantitatively relating statistical properties of Ly $\alpha$ absorption systems to statistical properties of galaxies.

\section{Constraints on the Unseen Galaxy Population}

The predicted number density $n(z)$ of Ly $\alpha$ absorption systems that arise in extended gaseous envelopes of galaxies is

$$
n(z)=\frac{c}{H_{0}}(1+z)\left(1+2 q_{0} z\right)^{-1 / 2} \int_{L_{B_{\min }}}^{\infty} d L_{B} \Phi\left(L_{B}, z\right) \pi R^{2}\left(L_{B}\right),
$$

where $c$ is the speed of light, $H_{0}$ is the Hubble constant, $z$ is the redshift $\Phi\left(L_{B}, z\right)$ is the galaxy luminosity function, $R\left(L_{B}\right)$ is the galaxy gaseous radius, and $L_{B_{\min }}$ is the minimum galaxy luminosity under consideration. By adopting the known (from equation 1) relationship between galaxy gaseous radius and galaxy $B$-band luminosity and a known galaxy luminosity function, comparison of the predicted and observed number densities of Ly $\alpha$ absorption systems constrains "unseen" galaxies that are not represented by the galaxy luminosity function.

Results of this comparison are shown in Figure 4. Figure 4 shows the observed number density of Ly $\alpha$ absorption systems (solid circles) and the predicted number density of Ly $\alpha$ absorption systems (open circles), based on a galaxy luminosity function determined from photometric redshifts of galaxies in the Hubble Deep Field (Fernández-Soto, Lanzetta, \& Yahil 1998). Figure 4 also shows the predicted number density of Ly $\alpha$ absorption systems corrected for incompleteness at faint galaxy luminosities (crosses). The primary result of Figure 4 is that to within measurement error known galaxies of known gas cross sections can account for all Ly $\alpha$ absorption systems at redshifts $z<2$ (and for most or all Ly $\alpha$ absorption systems at higher redshifts, after allowing for incompleteness at faint galaxy luminosities.) This suggests that the "unseen" galaxy 


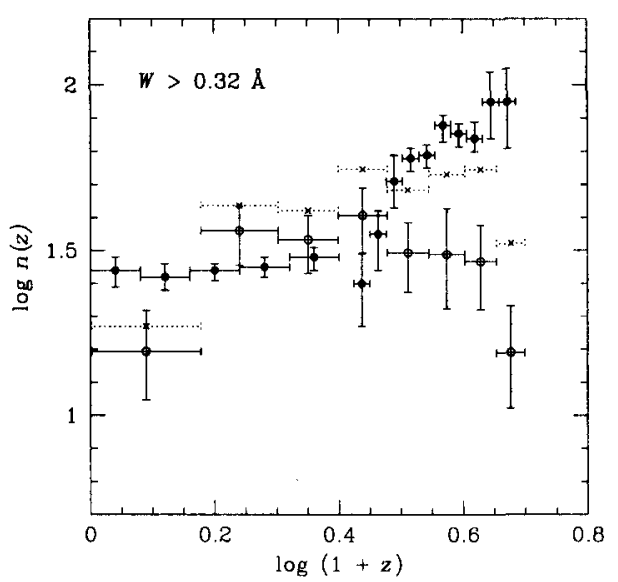

Figure 4. Observed (solid circles) and predicted (open circles) number densities of Ly $\alpha$ absorption sytems. Observed measurements are taken from Bechtold (1994) and Weymann et al. (1998). Crosses show predicted number density of Ly $\alpha$ absorption systems corrected for incompleteness at faint galaxy luminosities.

population produces at most a small fraction of the Ly $\alpha$ absorption systems. For the scaling relationship of equation (1) with $t=0.4$, equation (4) indicates that the number density of Ly $\alpha$ absorption systems is roughly proportional to the $B$-band luminosity density of the universe (multiplied by weakly redshiftdependent factor). We thus conclude that any "unseen" low surface brightness galaxies are unlikely to contribute significantly the the luminosity density of the universe.

Acknowledgments. This research was supported by NASA grant NAGW4422 and NSF grant AST-9624216.

\section{References}

Barcons, X., Lanzetta, K. M., \& Webb, J. K. 1995, Nature, 376, 321

Bechtold, J. 1994, ApJS, 91, 1

Chen, H.-W., Lanzetta, K. M., Webb, J. K., \& Barcons, X. 1998, ApJ, 498, 77

Fernández-Soto, A., Lanzetta, K. M., \& Yahil, A. 1998, AJ, in press

Lanzetta, K. M., Webb, J. K., \& Barcons, X. 1997, in Proc. of the 18th Texas

Symp. on Relativistic Astrophysics, ed. A. Olinto, J. Frieman, \& D.

Schramm, in press

Lanzetta, K. M., Bowen, D. V., Tytler, D., \& Webb, J. K. 1995, ApJ, 442, 538

Weymann, R. J., et al. 1998, ApJ, in press 


\section{Searching for LSB - II}

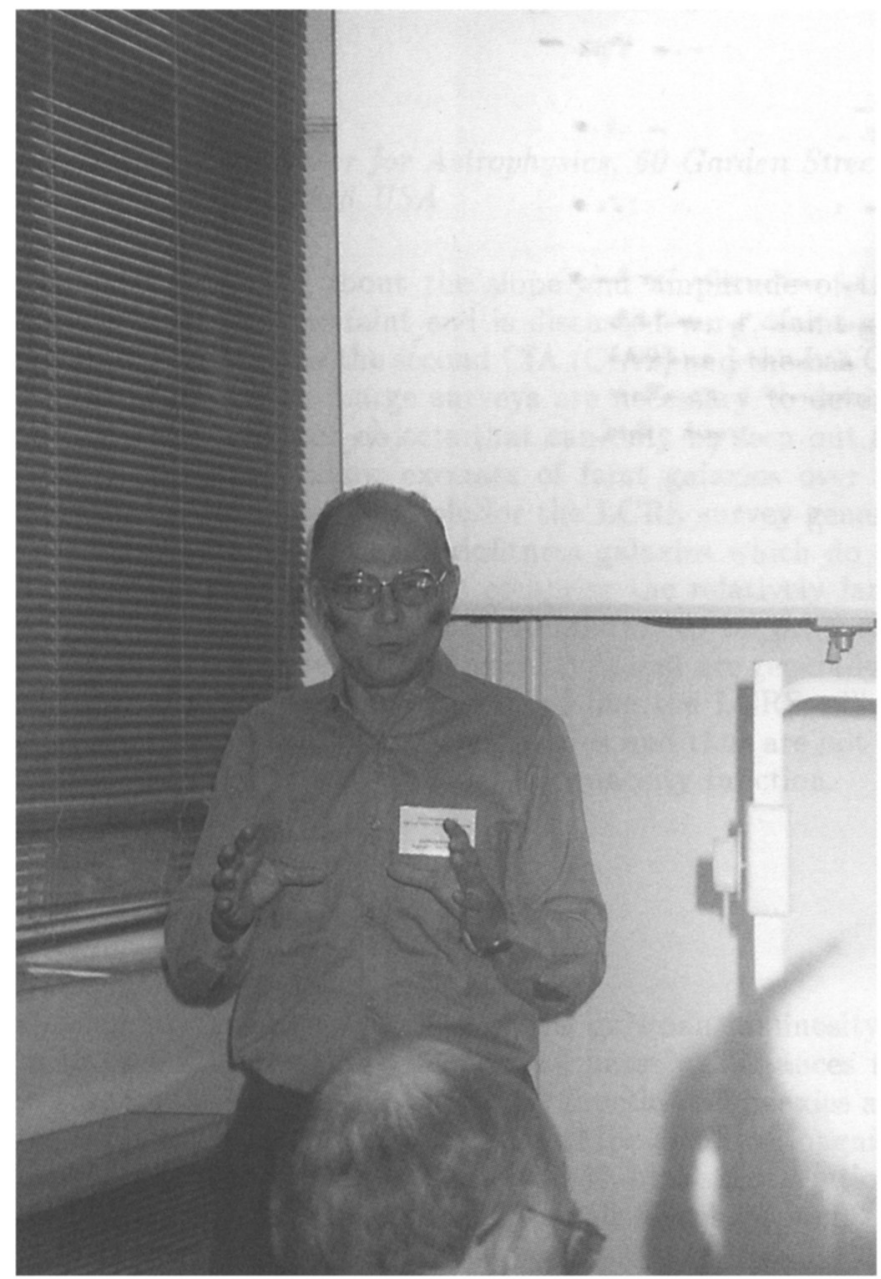

It's here, can't you see, between my hands. 